\title{
Isolation of adenovirus type 19 from the male and female genital tracts
}

\author{
GERALD B HARNETT* AND WILLIAM A NEWNHAM† \\ From the *Virus Laboratory, State Health Laboratory Services, and the + Public Health Venereal Disease \\ Control Centre, Perth, Western Australia
}

SUMMARY During routine screening for genital herpes simplex virus infection in patients attending a sexually transmitted diseases clinic adenovirus type 19 was isolated from both men and women. Peak incidences of genital infection with adenovirus type 19 corresponded with those of eye infection with the same virus in the general community. Thus, the relationship between genital and eye infection with adenovirus, the part played by genital infection in its dissemination, and the clinical symptoms it may produce need further study.

\section{Introduction}

In 1977 some cases of eye infection due to adenovirus type 19 occurred in Perth, Western Australia; this was the first time this serotype had been noted in the area, although since 1968 eye swabs have been tested in cell cultures appropriate for the isolation of adenovirus type 19.

During and after this outbreak adenovirus type 19 was isolated from urethral and cervical specimens collected from male and female patients attending a clinic for sexually transmitted diseases in Perth. In two patients with eye infection adenovirus type 19 was isolated from both the conjunctiva and genital tract.

This study reports the incidence of adenovirus type 19 in Perth, Western Australia, and discusses the possible implications of its presence in the genital tract.

\section{Patients and methods}

\section{SPECIMENS}

Specimens from patients with eye disease were submitted from various hospitals, eye specialists, and medical practitioners in Western Australia; these were conjunctival swabs broken off into a virus transport medium of Hanks' balanced salt solution with $0.5 \%$ bovine albumin, $0.07 \%$ sodium bicarbonate, and $50 \mu \mathrm{g} / \mathrm{ml}$ of gentamicin.

Address for reprints: Mr G B Harnett, Virus Laboratory, State Health Laboratory Services, Queen Elizabeth II Medical Centre, PO Box F312, Perth, Western Australia 6001

Accepted for publication 4 July 1980
Specimens from the genital tract were collected from men and women mainly attending the Public Health Venereal Disease Control Centre in Perth, Western Australia. They were collected routinely for isolation of chlamydia and herpes simplex virus. Urethral and cervical swabs were broken off into sucrose-phosphate transport medium containing $50 \mu \mathrm{g} / \mathrm{ml}$ each of vancomycin and streptomycin.

\section{CULTURE}

All specimens were inoculated into one tube of a line of human embryonic lung diploid fibroblasts of local derivation (HF-32). Cell cultures of HF-32 were grown in Eagle's MEM (Commonwealth Serum Laboratories, Melbourne, Australia) with $10 \%$ fetal calf serum, $0 \cdot 14 \%$ sodium bicarbonate, $28 \mathrm{mmol}$ $\mathrm{N}$-2-hydroxyethylpiperazine- $\mathrm{N}$-2-ethanesulphonic acid (HEPES), and 100 units/ml penicillin, $100 \mu \mathrm{g} / \mathrm{ml}$ streptomycin, and $35 \mu \mathrm{g} / \mathrm{ml}$ neomycin. After inoculation the same medium was used but with $2 \%$ fetal calf serum. HF-32 cultures were kept for 21 days at $33^{\circ} \mathrm{C}$ in a roller apparatus before being discarded as negative; cultures were examined for cytopathic effect (CPE) twice or three times weekly. In 47 positive cases, where data were adequately recorded, CPE took a mean time of $11 \cdot 3$ days to appear. From the results of specimens that were inoculated into primary monkey kidney, Vero, FL Amnion, or L 132 cell cultures in addition to HF-32 it was clear that HF-32 was the most sensitive cell line for the isolation of adenovirus type 19.

\section{IDENTIFICATION}

Initial isolates of adenovirus type 19 were identified by a neutralisation test with reference equine 
antiserum supplied by the World Health Organisation (WHO). Later isolates were identified using a neutralising antiserum, which was made available by $\operatorname{Dr} P$ A Phillips. Other adenovirus serotypes were identified using WHO or commercially supplied antisera (Grand Island Biological Company, New York 14072; Microbiological Associates, Bethesda, Maryland, 20016). Certain initial isolates were also examined by electron microscopy and showed typical adenovirus morphology. Adenovirus type 19 was reisolated from a representative sample of specimen aliquots stored over liquid nitrogen to show that the virus had not been introduced into cell cultures during laboratory procedures.

\section{Results}

During the study period between January 1977 and September 1979, 5923 respiratory tract specimens, 1201 eye specimens, and 30072 genital specimens were submitted for viral isolation. Adenovirus type 19 was isolated from two specimens from the respiratory tract, from 39 from the eye, from 34 from the male urethra, and from $\mathbf{2 5}$ from the female cervix (table). Other viruses isolated from the genital tract

TABLE Adenovirus serotypes isolated from the respiratory tract, eye, urethra, and cervix between January 1977 and September 1979

\begin{tabular}{lrrrr}
\hline \multicolumn{4}{c}{ No of adenoviruses isolated } \\
\cline { 2 - 5 } $\begin{array}{l}\text { Adenovirus } \\
\text { serotype }\end{array}$ & $\begin{array}{l}\text { Respiratory } \\
\text { tract }\end{array}$ & Eye & Urethra & Cervix \\
\hline 1 & 26 & 1 & 0 & 0 \\
2 & 58 & 5 & 0 & 3 \\
3 & 37 & 27 & 0 & 0 \\
5 & 19 & 0 & 0 & 0 \\
7 & 44 & 22 & 0 & 0 \\
8 & 0 & 10 & 0 & 0 \\
9 & 1 & 5 & 0 & 0 \\
14 & 2 & 0 & 0 & 0 \\
15 & 1 & 0 & 0 & 0 \\
19 & 2 & 39 & 34 & 25 \\
21 & 0 & 1 & 0 & 0 \\
Untyped & 2 & 6 & 3 & 3 \\
No of specimens tested & 5923 & 1201 & 17504 & 12568 \\
\hline
\end{tabular}

were adenovirus type 2 (three isolations) and untyped adenoviruses (three isolations). Of 59 patients from whom adenovirus type 19 was isolated from the genital tract, two with conjunctivitis also had eye infection with adenovirus type 19, and in a further four conjunctivitis was noted on their clinical cards but conjunctival swabs were not collected. Retrospective analysis of the clinical cards for the remaining patients indicated no history of eye disease, but this was not routinely noted unless the patients themselves mentioned it. Of 34 men with urethral swabs giving positive results for adenovirus type 19, 13 had nongonococcal urethritis; chlamydia group A or other agents known to cause nongonococcal urethritis were not isolated. The monthly distribution of adenovirus isolations for the study period, January 1977 to September 1979 inclusive, is shown in the figure.

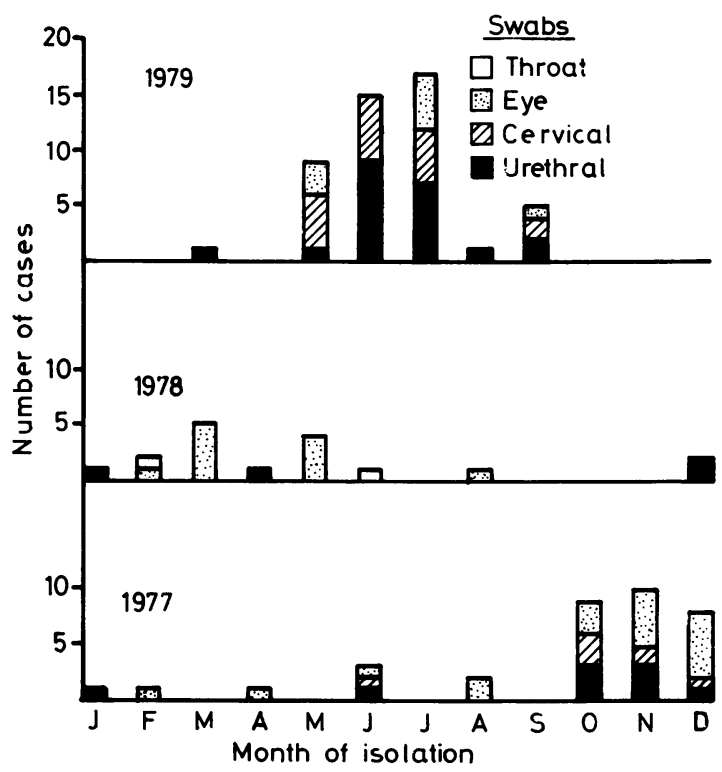

FIG Monthly isolations of adenovirus type 19 between January 1977 and September 1979 by the State Health Virus Laboratory of Western Australia.

The persistence of adenovirus type 19 in the genital tract was shortlived; of 16 patients with cervical or urethral swabs giving positive results for adenovirus type 19 who were resampled after a mean of 13.5 days, only one (a woman) still yielded adenovirus nine days after the initial positive cervical swab was collected.

\section{Discussion}

Adenovirus type 19 was first isolated in $1955^{1}$ and outbreaks of conjunctivitis and keratoconjunctivitis caused by it have occurred in the USA and Europe since $1973 . .^{2-4}$ In some of these outbreaks eye infection with adenovirus type 8 was also seen; 10 such cases were detected in our study. Conjunctivitis and keratoconjunctivitis due to adenovirus type 19 were first detected in Perth early in 1977, most cases being seen in October, November, and December. The number of cases then declined but a further peak occurred from Mav 1979 onwards. 
Coincidental with the peak incidences of eye disease due to adenovirus type 19 in the general community, we detected a corresponding peak incidence of genital tract infection with adenovirus type 19 in male and female patients attending the STD clinic in Perth. This finding occurred fortuitously during the routine investigation of these patients for infection with chlamydia and herpes simplex virus. Thirteen of the $\mathbf{3 4}$ male patients with adenovirus type 19 infection of the urethra attended the clinic because of nongonococcal urethritis (NGU); no chlamydia or other agents causing NGU were detected in these 13 patients, but the data are insufficient to associate adenovirus type 19 infection with NGU.

Although adenoviruses are frequently found to infect mucous membranes, and adenovirus type 11 has been isolated from the urine of children with haemorrhagic cystitis, ${ }^{5}$ we have found only one previous account of adenovirus isolation from the genital tract. Laverty et $a l^{6}$ reported the isolation of adenovirus type 19 from the cervix of a woman with conjunctivitis, sore throat, cervical lymphadenopathy, and paresthesia of the legs. Naib ${ }^{7}$ found abnormal cells suggesting adenovirus infection in vaginal smears from four patients.

Apart from three isolations of adenovirus type 2 and three of adenoviruses so far not typed, adenovirus 19 was the only serotype that we found in the genital tract, although many other serotypes were isolated from other anatomical sites in other patients during the study period. It may be that adenovirus type 19 has a special predilection for the genital tract similar to that apparently possessed by adenovirus type 11 for the urinary tract, and its presence in the genital tract raises the possibility of transmission by sexual contact.

An epidemiological study of an outbreak of eye disease due to adenovirus type 19 and type 8 showed that intrafamilial spread was commonplace and that spread in factories could be linked to the use of infected towels; also there was interfamilial spread of adenovirus type 19 among young adults. ${ }^{3}$ If adenovirus type 19 can be spread by sexual contact, it could perhaps cause inapparent infection in some patients before leading to eye infection by selfinoculation. As there was only a low incidence of eye infection in the patients with genital infections due to adenovirus type 19 , it seems unlikely that eye infection usually precedes genital infection.

It is plausible to suggest, however, that adenovirus type 19 can cause a pattern of oculogenital infection similar to that of chlamydia group A. In this study, genital infection with adenovirus seemed to be transient, but further studies are required to examine its persistence and to determine whether or not latent infection occurs. Further studies are also required to define the relationship between genital and eye infection with adenovirus, the part played by genital infection in the dissemination of adenovirus type 19, and the clinical symptoms it may produce.

We wish to thank the staff of the Public Health Venereal Disease Control Centre of Western Australia and of the State Health Virus Laboratory for the clinical, clerical, and laboratory work connected with this study; Miss C Jezewski, for preparation of the manuscript; Professor C A Mimms, who encouraged the publication of this work and read the manuscript; and Dr J C McNulty, Commissioner of Public Health and Medical Services, Western Australia, for permission to publish our findings.

\section{References}

1. Bell SD jun, McComb DE, Murray ES, Shih-man Chang R, Snyder JC. Adenoviruses isolated from Saudi Arabia. Epidemiologic features. Am J Trop Med Hyg 1959; 8:492-500.

2. Guyer B, O'Day DM, Hierholzer JC, Schaffner W. Epidemic keratoconjunctivitis: a community outbreak of mixed adenovirus type 8 and type 19 infection. $J$ Infect Dis 1975; 132: $142-50$

3. Vastine DW, West CE, Yamashiroya $\mathrm{H}$, et al. Simultaneous nosocomial and community outbreak of epidemic keratoconjunctivitis with types 8 and 19 adenovirus. Trans Am Acad Ophthalmol Otolaryngol 1976;81:826-40.

4. Darougar S, Quinlan MP, Gibson JA, Jones BR, McSwiggan DA. Epidemic keratoconjunctivitis and chronic papillary conjunctivitis in London due to adenovirus type 19. $B r J$ Ophthalmol 1977;61:76-85.

5. Numazaki $\mathrm{Y}$, Shigeta $\dot{\mathrm{S}}$, Kumasaka $\mathrm{T}$, et al. Acute hemorrhagic cystitis in children. Isolation of adenovirus type 11. N Engl J Med 1968;278:700-4.

6. Laverty CR, Russell P, Black J, Kappagoda N, Benn MB Booth N. Adenovirus infection of the cervix. Acta Cyto (Baltimore) 1977;21:114-7.

7. Naib ZM. Exfoliative cytology of viral cervico-vaginitis. Acto Cytol (Baltimore) 1966; 10:126-9. 